\title{
Viscoelastic Relaxation of Cross-Linked, Alternating Copolymers in the Free-Draining Limit
}

\section{Cristian Satmarel, ${ }^{\dagger}$ Andrew A. Gurtovenko, ${ }^{\dagger, \ddagger}$ and Alexander Blumen*,†}

Theoretische Polymerphysik, Universität Freiburg, Hermann-Herder-Strasse 3, D-79104 Freiburg, Germany, and Institute of Macromolecular Compounds, Russian Academy of Sciences, Bol shoi Prospect 31, V.O., St. Petersburg, 199004 Russia

Received September 6, 2002; Revised Manuscript Received November 15, 2002

\begin{abstract}
We study theoretically the viscoelastic relaxation of cross-linked copolymers in the framework of generalized Gaussian structures (GGS), which are extensions of the Rouse model to arbitrary geometries. The model holds for general homogeneous copolymers under free-draining conditions; it is especially suitable for treating alternating copolymers. We calculate the storage $\mathrm{G}^{\prime}(\omega)$ and the loss $\mathrm{G}^{\prime \prime}(\omega)$ moduli both for un-cross-linked and for cross-linked copolymer chains, and observe a variety of features: al ternating copolymers differ from homopolymers in the high-frequency domain, where $G^{\prime \prime}(\omega)$ may di splay two maxima. Cross-linking alternating A-B copolymer chains into regular networks (lattices) leads to the appearance of a network-dominated, low-frequency relaxation domain. In the case of very large differences in the mobility of the A monomers, of the B monomers, and of the cross-links, $G^{\prime}(\omega)$ is very structured, displaying three relaxation domains, separated by two plateaus. G" $(\omega)$ shows three peaks. We expect that these features can be readily detected through appropriate mechanical relaxation experiments.
\end{abstract}

\section{Introduction}

In this work we study the influence of the presence of different types of monomers, (e.g., A and B) on the dynamical properties of polymeric networks. By this, we continue our investigations of the possibilities offered by the so-called generalized Gaussian structures $(G G S)^{1-8}$ in the study of polymeric materials. GGS extend the Rouse model $^{9-11}$ to arbitrary geometries. The basic idea behind these Gaussian approaches consists of using a coarse-grained description of the polymer based on beads, which are connected to each other by (entropic) springs. Distinct from the classical Rouse-model, initially developed for linear chains, GGS allow some of the beads to be multifunctional; such beads are then connected to more than two neighbors and represent construction elements that lead to extended networks. In this work, we enlarge the analysis of the mechanical relaxation of such networks by also treating systems made up from different monomer species. Such theoretical considerations were performed for linear block copolymers in a pioneering series of papers ${ }^{12-15}$ about three decades ago, see also refs 16 and 17. As a first step, we will stay in the framework of free-draining structures, by not (yet) taking hydrodynamic interactions $^{18}$ into account. Also not considered are specific monomer-monomer interactions (such as the excludedvolume or the attraction or repulsion between monomers of different kinds). As we proceed to show, al ready this simplified GGS treatment leads to a wealth of interesting features in the relaxation patterns.

We would like to note from the start that copolymers are a very important class of materials and that crosslinked copolymer systems are of particular interest, because they combine the properties of copolymer materials with those of polymer networks, leading to

* To whom correspondence should be addressed.
† Universität Freiburg.

₹ Russian Academy of Sciences. viscoelastic materials with a wide range of technological applications. Some of their unique properties are related to the separation of block copolymers on microscopic scales, ${ }^{19-21}$ a feature which our version of the GGS model does not account for. However, one al so encounters homogeneous copol ymers, by which one means that they do not microphase separate. As examples, we mention here block copolymers of styrene and $\alpha$-methylstyrene $22-25$ and polystyrene-block-polyisopropene-bl ockpolystyrene copolymers. ${ }^{26}$ Homogeneity can also be achieved by the proper choice of the solvent. 27,28

In this paper, we apply the GGS model to cross-linked copolymers. In general, one has two types of networks. First, there are physical (transient) networks of gelforming copolymer solutions. ${ }^{29-31}$ The second type of networks are those attained through chemical reactions, leading to the formation of permanent cross-links, ${ }^{32-34}$ such as the copolymerization of styrene with ethylene glycol dimethacrylate. ${ }^{35}$

In this work, we focus on the case of permanent crosslinks. In fact, the proposed approach can also be applied to transient networks (gels) as long as one measures on time scales smaller than the average lifetime of the cross-links in the gel. As models for copolymer networks, we consider regular lattices built from copolymer chains. In so doing, we general ize some previous works rel ated to polymer networks. ${ }^{6-8}$ We work exemplarily with networks made of copolymer chains, with alternating beads of $-A B A B$ - type, the motivation being that they are structures of much interest ${ }^{36-42}$ and that for them the question of microphase separation-evidently-does not arise.

The paper is organized as follows: in the next section, we devel op the mathematical model on which all further considerations are based, paying particular attention to the modeling of the different constituents (monomers) of the network. In section 3, we focus on the relaxation spectrum of regularly built networks made from copolymer chains. The next two sections are devoted to the 
(a)

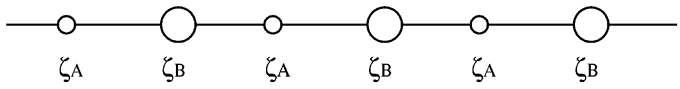

(b)

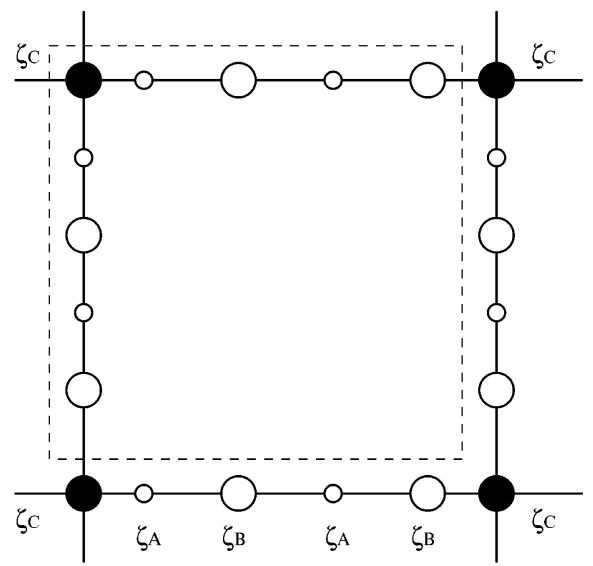

Figure 1. Polymer systems under study: (a) an alternating copolymer chain containing two kinds of beads; (b) an elementary cell (dashed line) of a square lattice built from such (identical) copolymer chains.

application of the formalism to chains made of alternating beads. Section 4 deals with the mechanical relaxation of linear, un-cross-linked alternating chains, a problem which can be treated fully analytically, and which was previously only numerically solved. ${ }^{12}$ Section 5 discusses the effects which arise from cross-linking such alternating chains into regular lattices. In section 6 , we end with a short summary and conclusions.

\section{The Model}

We focus here on the dynamic behavior of polymer networks, whose main constituents are $A$ and $B$ monomers. In this way the network is built from -AAAAand - BBBB - segments such that an A- segment is connected to a B segment and viceversa. Furthermore, there also exist multifunctional centers (e.g., crystallites ${ }^{31}$ ) to which more than two segments are attached; these centers are fundamental in the formation of the network. For simplicity, we assume first that all -AAAAsegments are similar (i.e., have roughly the same length) and that the same holds true for the -BBBBsegments. Then we can, in a coarse-grained description, go over to view each of these segments as one bead, i.e., we have $A$ beads and $B$ beads, respectively. This coarsegrained procedure leads to alternating copolymers of $A$ and $B$ beads. A simple situation is presented in Figure 1 , in which in part a we indicate such an alternating, copolymer model chain. In part b of Figure 1, we have drawn a two-dimensi onal regular network consisting of alternating copolymer chains, the junction points being multifunctional beads of friction constant $\xi_{c}$. Given also the different nature of the -AAAA - and -BBBBsegments, the $A$ and $B$ beads differ in their dynamical properties; we will focus here on the possibility that they have different friction constants, $\zeta_{\mathrm{A}}$ and $\xi_{\mathrm{B}}$. On the other hand, for simplicity, we take all spring constants $K$ of the model to be equal. All these features are in line with the classical work by Hansen and Shen, ${ }^{12}$ who also focused in their study of copolymer blocks on such differences in the frictional coefficients: Hansen and Shen considered situations in which the ratio $\xi_{A} / \xi_{B}$ may be as large as 10 000; some of their analysis centered on the $\xi_{A} / \zeta_{B}$ value of 185 , corresponding to the friction coefficients of polystyrene and poly( $\alpha$-methylstyrene). ${ }^{12}$ In this work, we will also span such large ranges of $\xi$ values.

Now, let us outline the measurable relaxation quantities which we are interested in and which are experimentally accessible. In general, mechanical relaxation is a well-established technique in polymer physics. In typical mechanical experiments one measures the compl ex (shear) modulus $\mathrm{G}^{*}(\omega)$, as response to a harmonic strain field. In the framework of the Rouse model, refs 10 and 43 show that for homopolymer chains the foll owing standard expressions for $\mathrm{G}^{\prime}(\omega)$, the real, and for $\mathrm{G}^{\prime \prime}(\omega)$, the imaginary component of $\mathrm{G}^{*}(\omega)$, hold:

$$
\left[\mathrm{G}^{\prime}(\omega)\right]=\frac{1}{\mathrm{~N}_{\text {tot }}} \sum_{\mathrm{i}=2}^{\mathrm{N}_{\text {tot }}} \frac{\left(\omega \tau_{\mathrm{i}}\right)^{2}}{1+\left(\omega \tau_{\mathrm{i}}\right)^{2}}
$$

and

$$
\left[G^{\prime \prime}(\omega)\right]=\frac{1}{N_{\text {tot }}} \sum_{i=2}^{N_{\text {tot }}} \frac{\omega \tau_{\mathrm{i}}}{1+\left(\omega \tau_{\mathrm{i}}\right)^{2}}
$$

where $\mathrm{N}_{\text {tot }}$ is the total number of elements (beads) in the system under study and $\tau_{\mathrm{i}}$ are the $\left(\mathrm{N}_{\text {tot }}-1\right)$ finite relaxation times of the system. Here we will use the mechanical relaxation functions in the form of the reduced storage and loss moduli, [G' $(\omega)]$ and $\left[\mathrm{G}^{\prime \prime}(\omega)\right]$, given that we are mostly interested in the shapes of $\mathrm{G}^{\prime}(\omega)$ and $\mathrm{G}^{\prime \prime}(\omega)$, but not in their prefactors. In such a general form, eqs 1 and 2 are equally applicable to very dilute solutions as well as to concentrated ones (at least when entangl ement effects are negligible). ${ }^{10,43}$

It is generally accepted, ${ }^{14,17}$ based on linear-response theory and on thermodynamic arguments that eqs 1 and 2 for the storage and loss moduli hold in general; they hence should be unaffected by polymer chain composition, such as having beads with different friction constants. In other words, the standard relations given by eqs 1 and 2 hold al so for copolymer systems, and the heterogeneity enters only through the relaxation times. ${ }^{14,17}$ By this, the problem of determining the mechanical relaxation of copolymers is reduced to the eigenvalue problem from which the relaxation times $\tau_{\mathrm{i}}$ follow.

On the basis of the expressions of ref 10 for the Rouse model, in the Langevin framework generalized to our situation here, the position vector $\mathbf{R}_{\mathbf{i}}(\mathrm{t})$ of the ith bead of a copolymer network obeys ${ }^{1,5,6,7,44}$

$$
\zeta_{i} \frac{d \mathbf{R}_{i}(t)}{d t}+K \sum_{j=1}^{N_{\text {tot }}} A_{i j} \mathbf{R}_{j}(t)=\mathbf{f}_{i}
$$

where $\xi_{i}$ is the friction constant of the ith bead, $\mathbf{f}_{i}$ the sum of stochastic forces acting on it, and $\mathbf{A}=\left(\mathrm{A}_{\mathrm{ij}}\right)$ is the connectivity matrix of the network; see ref 1 and 44 for details. The nondiagonal $A_{i j}$ equals -1 if the ith and $j$ th beads are connected and 0 otherwise; $A_{i i}$ equals the number of bonds emanating from the ith bead. Furthermore, the sets $\left\{\xi_{i}\right\}$ and $\left\{\mathbf{f}_{i}\right\}$ are connected via the fluctuation-dissipation theorem. Given, however, that we are interested only in the eigenfrequencies of the system, which depend solely on $\mathbf{A}$, we do not enter into these details. It is well-known that in the frame work of the Rouse model it is sufficient to focus on the thermally averaged Cartesian components of $\left\{\mathbf{R}_{\mathbf{i}}\right\}$ in 
order to get the relaxation spectrum. ${ }^{9,10,14}$ From now on we will hence view eq 3 as relating to thermally averaged variables, under which averaging its righthand-side vanishes. We note, furthermore, that eq 3 can describe the dynamics of geometric patterns which are much more complex ${ }^{1}$ than what is shown in Figure 1. Evidently, incorporating more complex chains creates no problems, the regular pattern of Figure 1 ensuring that the GGS dynamics can be computed to high accuracy, ${ }^{6-8}$ as we recall in the following.

We now rewrite the above set of Langevin equations, to recast it into a form similar to that which holds for a homopolymer system (where for all beads $\xi_{i}$ has the same value). For that we consider beads of the first kind $\left(\xi_{A}\right)$ as reference and have as their characteristic relaxation time $\tau_{0}=\zeta_{\mathrm{A}} / \mathrm{K}$. For the other kinds of beads we set

$$
\sigma_{\mathrm{z}}=\frac{\zeta_{\mathrm{A}}}{\zeta_{\mathrm{z}}}
$$

In this way and in the spirit discussed above, we have for the thermally averaged $\mathbf{R}_{\mathrm{i}}(\mathrm{t})^{12,13,14,16,17}$

$$
\frac{\mathrm{d} \mathbf{R}_{\mathrm{i}}(\mathrm{t})}{\mathrm{dt}}+\left(1 / \tau_{0}\right) \sum_{\mathrm{j}=1}^{\mathrm{N}_{\text {tot }}} \tilde{\mathrm{A}}_{\mathrm{ij}} \mathbf{R}_{\mathrm{j}}(\mathrm{t})=0
$$

with

$$
\tilde{\mathrm{A}}_{\mathrm{ij}}=\sigma_{\mathrm{z}_{\mathrm{i}}} \mathrm{A}_{\mathrm{ij}}
$$

Here the matrix $\tilde{\mathbf{A}}=\left(\tilde{A}_{i j}\right)$ is obtained from $\mathbf{A}$ by multiplying its rows with the $\sigma_{\mathrm{z}_{\mathrm{i}}}$ corresponding to the different molecular species $z_{i}$. Then $\tilde{\mathbf{A}}$ is, in general, not symmetric anymore. In this way, the copolymer system under study differs from a homopolymer system.

However, the eigenvalues of $\tilde{\mathbf{A}}$ are still real and nonnegative. This is seen by noticing from eq 6 that $\tilde{\mathbf{A}}$ can be written as

$$
\tilde{\mathbf{A}}=\mathbf{S A}
$$

where the matrix $\mathbf{S}$ is diagonal, its nonvanishing el ements being $\mathrm{S}_{\mathrm{ii}}=\sigma_{\mathrm{z}_{\mathrm{i}}}>0$. It follows that all related matrices such as $\mathbf{S}^{-1}, \mathbf{S}^{1 / 2}$, and $\mathbf{S}^{-1 / 2}$ exist and are diagonal. We focus now on the matrix $\mathbf{T}=\mathbf{S}^{1 / 2} \mathbf{A} \mathbf{S}^{1 / 2}$. Evidently, $\mathbf{T}$ is symmetric. Moreover, $\mathbf{A}$ is positivedefinite, $\sum_{i j} x_{i} A_{i j} x_{j} \geq 0$ for all vectors $\mathbf{X}=\left(x_{1}, \ldots, x_{N_{\text {tot }}}\right)^{\top}$. Hence one also has $\sum_{i j} x_{i} \sqrt{\sigma_{z_{i}}} A_{i j} \sqrt{\sigma_{z_{j}}} x_{j} \geq 0$ for all vectors $\mathbf{X}$, which means that $\mathbf{T}=\mathbf{S}^{1 / 2} \mathbf{A} \mathbf{S}^{1 / 2}$ is also positive definite. Hence a unitary transformation $\mathbf{Q}$ which diagonalizes $\mathbf{T}$ exists

$$
\mathbf{Q}^{-1} \mathbf{T} \mathbf{Q}=\mathbf{\Lambda}
$$

where the diagonal values $\lambda_{\mathrm{i}}$ of $\boldsymbol{\Lambda}$ are real and nonnegative. From eq 8 it follows, by introducing $\mathbf{S}^{-1 / 2} \mathbf{S}^{1 / 2}$ $=\mathbf{1}$ :

$$
\mathbf{\Lambda}=\mathbf{Q}^{-1} \mathbf{1} \mathbf{S}^{1 / 2} \mathbf{A S}^{1 / 2} \mathbf{Q}=\mathbf{Q}^{-1} \mathbf{S}^{-1 / 2} \mathbf{S A S}^{1 / 2} \mathbf{Q}
$$

Setting $\mathbf{S}^{1 / 2} \mathbf{Q}=\tilde{\mathbf{Q}}$ one thus has $\tilde{\mathbf{Q}}^{-1} \tilde{\mathbf{A}} \tilde{\mathbf{Q}}=\mathbf{\Lambda}$, i.e., $\tilde{\mathbf{Q}}$ diagonalizes $\tilde{\mathbf{A}}$, and all eigenvalues $\lambda_{i}$ of $\tilde{\mathbf{A}}$ are real and nonnegative. From these $\lambda_{\mathrm{i}}$ the relaxation times $\tau_{\mathrm{i}}$ needed in the eqs 1 and 2 follow in a very simple fashion:

$$
\tau_{\mathrm{i}}=\tau_{0} / 2 \lambda_{\mathrm{i}}
$$

Note that the factor 2 in the relaxation times in eq 10 arises from the second moment of the displacements involved in computing the stress, see the corresponding derivation of the expressions for the moduli in ref 10 .

\section{General Method}

In this section, we display a general approach to treat the dynamics of regular lattices whose vertexes are connected by identical linear copolymer chains; these have thus an arbitrary sequence of beads of different kinds. This regularity allows us, based on previous works, ${ }^{6-8}$ to simplify the problem using Floquet's theorem (whose special case in solid-state physics is Bloch's theorem ${ }^{45-47}$ ).

We focus on a (topologically) two-dimensional square lattice. The elementary cell of the square lattice is denoted by a two-component index $\boldsymbol{\Omega}=(\alpha, \beta)$ where $\alpha$ and $\beta$ range from 1 to $\mathrm{N}$. Such a square cell contains a vertex (a network junction) and two identical copolymer chains of $\mathrm{N}_{\text {chain }}$ beads each; i.e., it consists of $\mathrm{N}_{\text {cell }}=$ $\left(2 \mathrm{~N}_{\text {chain }}+1\right)$ beads, which we number by the index $\mid \in$ $\left\{1 . . . \mathrm{N}_{\text {cell }}\right\}$. The whole network consists then of $\mathrm{N}_{\text {cell }} \mathrm{N}^{2}$ beads, numbered as $(I, \mathbf{\Omega}) \equiv(I, \alpha, \beta)$. In general, we are not restricted to only two kinds $A$ and $B$ of beads but may have various species; also the junctions may be occupied by a different kind of bead, e.g., C. Thus, an elementary cell (as well as the whole network) may contain $\mathrm{N}_{\text {sort }}$ distinct kinds of beads, Figure $1 \mathrm{~b}$. Given that now this information (originally about $\xi_{i}$ ) is included in the elastic term of eq 5 , we can proceed in the same way as for homopolymers, ${ }^{6-8}$ having only to keep in mind that now the matrix $\tilde{\mathbf{A}}$ is nonsymmetric.

We can now simplify the determination of the eigenvalues of $\tilde{\mathbf{A}}$ by making use of the topological symmetry of the matrix. Of course, the eigenvalues of $\tilde{\mathbf{A}}$ are real and nonnegative, on the general grounds discussed before. With help of the above notations, we rewrite the elastic term in the Langevin equations of motion (which is determined through the topology of the network and through the set of $\xi_{i}$ ) in the following way:

$$
\begin{aligned}
& \sum_{\mathrm{j}=1}^{\mathrm{N}_{\text {tot }}} \tilde{\mathrm{A}}_{\mathrm{ij}} \mathbf{R}_{\mathrm{j}}(\mathrm{t})=\sum_{\mathrm{m}=1}^{\mathrm{N}_{\text {cell }}} \sum_{\mathbf{\Omega}^{\prime}} \tilde{\mathrm{A}}_{1 \mathbf{\Omega} \mathrm{m} \mathbf{\Omega}^{\prime}} \mathbf{R}_{\mathrm{m} \mathbf{\Omega}^{\prime}}(\mathrm{t})= \\
& \sum_{\mathrm{m}=1}^{\mathrm{N}_{\text {cell }}} \tilde{\mathrm{A}}_{\mid \mathbf{\Omega} \mathrm{m} \boldsymbol{\Omega}} \mathbf{R}_{\mathrm{m} \boldsymbol{\Omega}}(\mathrm{t})+\sum_{\mathrm{m}=1}^{\mathrm{s}} \sum_{\substack{\boldsymbol{\Omega}^{\prime} \\
\boldsymbol{\Omega}^{\prime} \neq \mathbf{\Omega}}} \tilde{\mathrm{A}}_{\mid \mathbf{\Omega} \mathrm{m} \boldsymbol{\Omega}^{\prime}}, \mathbf{R}_{\mathrm{m} \mathbf{\Omega}^{\prime}}(\mathrm{t})
\end{aligned}
$$

where we let $\mathrm{i} \rightarrow(\mathrm{I}, \mathbf{\Omega})$ and $\mathrm{j} \rightarrow\left(\mathrm{m}, \mathbf{\Omega}^{\prime}\right)$. Because of obvious symmetries, we may set in eq $11, \tilde{B}_{\mathrm{Im}}^{\text {(int) }} \equiv \tilde{\mathrm{A}}_{\mathbf{\Omega} \mathrm{m} \Omega}$ and $\tilde{\mathrm{B}}_{\operatorname{lm}}^{(\text {ext) }}(\boldsymbol{\Delta}) \equiv \tilde{\mathrm{B}}_{\operatorname{lm}}^{(\text {ext) }}\left(\boldsymbol{\Omega}-\boldsymbol{\Omega}^{\prime}\right) \equiv \tilde{\mathrm{A}}_{\mid \boldsymbol{\Omega} \mathrm{m} \boldsymbol{\Omega}^{\prime}}$, which leads to

$$
\begin{aligned}
\sum_{\mathrm{i}=1}^{\mathrm{N}_{\text {tot }}(\mathrm{t})} \tilde{\mathrm{A}}_{\mathrm{ij}} \mathbf{R}_{\mathrm{j}}(\mathrm{t})=\sum_{\mathrm{m}=1}^{\mathrm{N}_{\text {cell }}} \tilde{\mathrm{B}}_{\mathrm{Im}}^{(\mathrm{int})} \mathbf{R}_{\mathrm{m} \boldsymbol{\Omega}}(\mathrm{t})+ \\
\sum_{\mathrm{m}=1}^{\mathrm{N}_{\text {cell }}} \sum_{\boldsymbol{\Delta}} \tilde{\mathrm{B}}_{\operatorname{lm}}^{(\mathrm{ext})}(\boldsymbol{\Delta}) \mathbf{R}_{\mathrm{m}(\boldsymbol{\Omega}-\boldsymbol{\Delta})}(\mathrm{t})
\end{aligned}
$$

where $\boldsymbol{\Delta}=\boldsymbol{\Omega}-\boldsymbol{\Omega}^{\prime}$ is the relative distance between the lattice cells $\boldsymbol{\Omega}$ and $\boldsymbol{\Omega}^{\prime}$, measured in units of number of cells. In eq 12 the matrix $\tilde{\mathbf{B}}^{(\mathrm{int})}=\left(\tilde{\mathrm{B}}_{\mathrm{Im}}^{\text {(int) }}\right)$ is the subset of $\left\{\tilde{A}_{i j}\right\}$ related to a given cell consisting of $\mathbf{N}_{\text {cell }}$ beads. In contrast, the matrix $\tilde{\mathbf{B}}^{(\mathrm{ext})}(\boldsymbol{\Delta})=\left(\tilde{\mathrm{B}}_{\mathrm{Im}}^{(\text {ext) }}(\boldsymbol{\Delta})\right)$ provides the intercell connections: The nonzero elements of the matrices $\tilde{B}_{\operatorname{lm}}^{(\text {ext) }}(\boldsymbol{\Delta})$ have as values negative integers; a 
value $(-b)$ for $\tilde{B}_{\operatorname{lm}}^{(e x t)}(\boldsymbol{\Delta})$ indicates that the total number of connections between bead I of cell $\boldsymbol{\Omega}$ and bead $\mathrm{m}$ of cell $\boldsymbol{\Omega}^{\prime}=\boldsymbol{\Omega}-\boldsymbol{\Delta}$ equals $b$. In the case of a square regular lattice, the sum over $\boldsymbol{\Delta}$ in eq 12 runs only over nearestneighbor cells. Thus, in the 2D square network the cell $\boldsymbol{\Omega}=(\alpha, \beta)$ is directly connected to its following four neighbors: $(\alpha-1, \beta),(\alpha+1, \beta),(\alpha, \beta-1)$, and $(\alpha, \beta+1)$; hence $\Delta \in\{(1,0),(-1,0),(0,1),(0,-1)\}$.

Because of the symmetry inherent in eq 12, the diagonalization of $\tilde{\mathbf{A}}$ is now quite straightforward. From a formal point of view, the situation is very close to solid state physics (Bravais lattice with a basis). However, one should note that in the arguments leading to eq 12 only the connectivity (a topological feature) enters. Hence, the networks considered here are not required to be translationally invariant. Here we have a network consisting of flexible subunits, whose conformations change widely under the underlying random Brownian forces, and hence differ much from cell to cell. Nevertheless, using eq 12, we can proceed quite formally: We apply periodic boundary conditions to the whole copolymer network and look for solutions to the Langevin equations of the form $\mathrm{m}^{6-8}$

$$
\begin{aligned}
\mathbf{R}_{\mathrm{I \Omega}}(\mathrm{t}) \equiv \mathbf{R}_{\mathrm{l} \alpha \beta}(\mathrm{t})= \\
\sum_{\mathrm{k}_{1}, \mathrm{k}_{2}} \mathbf{C}_{\mid \mathbf{k}} \exp \left(\mathrm{i}\left[\mathrm{k}_{1} \alpha+\mathrm{k}_{2} \beta\right]\right) \exp \left(-\lambda(\mathbf{k}) \mathrm{t} / \tau_{0}\right)
\end{aligned}
$$

In eq 13 , i is the imaginary unit, the $\mathbf{C}_{\mathbf{l} \mathbf{k}}$ are constants, and the $\mathbf{k}=\left(\mathrm{k}_{1}, \mathrm{k}_{2}\right)$ obey:

$$
\mathrm{k}_{1}=\frac{2 \pi \mathrm{m}_{1}}{\mathrm{~N}} \text { and } \mathrm{k}_{2}=\frac{2 \pi \mathrm{m}_{2}}{\mathrm{~N}}
$$

where the integers $m_{1}$ and $m_{2}$ range from 0 to $(N-1)$. F urthermore, the $\lambda(\mathbf{k})$ denote the different eigenvalues as functions of the parameter $\mathbf{k}$. Distinct from solidstate physics, however, here the two-component vector k simply counts the eigenvalues (modes), and is not related to a reciprocal wave vector.

Inserting eqs 13 and 14 into the Langevin equations of motion leads to

$$
\lambda(\mathbf{k}) \mathbf{C}_{\mid \mathbf{k}}=\sum_{m=1}^{N_{\text {cell }}} \tilde{B}_{\text {Im }}(\mathbf{k}) \mathbf{C}_{m \mathbf{k}}
$$

where the elements of the matrix $\tilde{\mathbf{B}}(\mathbf{k})=\left\{\tilde{B}_{\mid m}(\mathbf{k})\right\}$ are given by

$$
\tilde{\mathrm{B}}_{\operatorname{Im}}(\mathbf{k})=\tilde{\mathrm{B}}_{\operatorname{lm}}^{\text {(int) }}+\sum_{\boldsymbol{\Delta}} \tilde{\mathrm{B}}_{\operatorname{lm}}^{(\mathrm{ext})}(\boldsymbol{\Delta}) \exp (-\mathrm{i} \mathbf{k} \cdot \boldsymbol{\Delta})
$$

We remark that the matrices $\tilde{\mathbf{B}}(\mathbf{k})$ include now (besi des the friction constants) all the information concerning the topology (connectivity) inside each cell and also the way in which the cells are connected to each other. F or each realization of the set $\left\{\xi_{i}\right\}$ inside the unit cell there are $\mathrm{N}^{2}$ different $\tilde{\mathbf{B}}(\mathbf{k})$ matrices, in which the $\exp (-\mathbf{i} \mathbf{k} \cdot \boldsymbol{\Delta})$ terms describe the phase shifts between lattice cells. According to eq 15 the problem of finding the eigenvalues of $\tilde{\mathbf{A}}$, which is a $\left(\mathrm{N}_{\text {cell }} \mathrm{N}^{2}\right) \times\left(\mathrm{N}_{\text {cell }} \mathrm{N}^{2}\right)$ matrix, is now reduced to the related problem of determining the eigenvalues of $\mathrm{N}^{2}$ different $\left(\mathrm{N}_{\text {cell }} \times \mathrm{N}_{\text {cell }}\right)$ matrices.

We close this section by stressing that the derivation of eq 15 presented here is general and that it holds for arbitrary bead sequences $\left\{\zeta_{\mathrm{i}}\right\}$ of the copolymer chains involved. Moreover, the 2D square lattice considered here is only a particular example of a regular lattice. Our approach can be easily extended to other lattice types. For instance, all the results of this section are (given the corresponding, simple changes in eqs 13 and 16 and the comments after eq 12) also valid for a 3D cubic lattice built from copolymer chains, as well as for a copolymer chain with repeat units; to describe them, one simply has to take into account one more component, $k_{3}$, of the vector $\mathbf{k}$ (for a 3D lattice) or to ignore one component, i.e., to set $k_{2}=0$ (for a 1D lattice). Below we present an application of this general approach to linear alternating copolymers and to square networks cross-linked from such copolymer chains.

\section{Alternating Copolymer Chains}

At first we apply the general method displayed in the previous section to an alternating copolymer chain -ABAB -; see Figure 1a. As parameters $\sigma_{\mathrm{i}}$ we have here $\sigma_{\mathrm{A}}=1$ and $\sigma_{\mathrm{B}}=\xi_{\mathrm{A}} / \xi_{\mathrm{B}}$. The relaxation spectrum of such an alternating copolymer chain was evaluated previously, as a special case for alternating block copolymer chains, using numerical methods. ${ }^{12}$ In the terminology of the previous section, such a chain consists of $\mathrm{N}$ unit cells of the form AB. For alternating block copolymers,

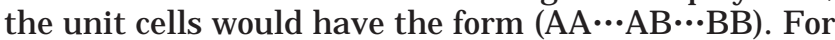
simplicity, we impose first periodic boundary conditions and close then the chain into a ring; by this we can apply directly the methods devel oped above.

According to eq 15 the problem of determining the eigenvalues of the alternating copolymer ring consisting of $2 \mathrm{~N}$ beads of two kinds ( $\mathrm{A}$ and $\mathrm{B}$ ) is reduced to the related problem for the $(2 \times 2)$ matrix $\tilde{\mathbf{B}}\left(k_{1}\right)$, which takes in our case the following form:

$$
\tilde{\mathbf{B}}\left(\mathrm{k}_{1}\right)=\left(\begin{array}{cc}
2 & -\left(1+\exp \left[-\mathrm{ik} \mathrm{k}_{1}\right]\right) \\
-\sigma_{\mathrm{B}}\left(1+\exp \left[\mathrm{ik}_{1}\right]\right) & 2 \sigma_{\mathrm{B}}
\end{array}\right)
$$

where $\mathrm{k}_{1}=2 \pi \mathrm{m}_{1} / \mathrm{N}$ with $\mathrm{m}_{1}=0, \ldots,(\mathrm{N}-1)$, see eq 14 . The relation $\operatorname{det}\left(\tilde{\mathbf{B}}\left(\mathrm{k}_{1}\right)-\lambda \mathbf{1}\right)=0$ leads to the following quadratic equation for the eigenvalues

$$
\lambda^{2}-2 \lambda\left(1+\sigma_{\mathrm{B}}\right)+2 \sigma_{\mathrm{B}}\left(1-\cos _{1}\right)=0
$$

from which the eigenvalues $\lambda$ follow:

$$
\lambda=\left(1+\sigma_{\mathrm{B}}\right) \pm \sqrt{\left(1+\sigma_{\mathrm{B}}\right)^{2}-2 \sigma_{\mathrm{B}}\left(1-\cos \mathrm{k}_{1}\right)}
$$

One can distinguish heretwo groups of eigenvalues. The first group (with $k_{1} \neq 0$ ) contains $(2 N-2)$ ei genvalues and involves displacements of the $A B$ unit cells with respect to each other. The second group (for $k_{1}=0$ ) has two eigenvalues, namely $\lambda=0$ and $\lambda_{1}=2\left(1+\sigma_{\mathrm{B}}\right)$. The eigenvalue $\lambda=0$ corresponds to the displacement (under the influence of the fluctuating forces) of the copolymer ring as a whole. The eigenvalue $\lambda_{1}=2\left(1+\sigma_{\mathrm{B}}\right)$ arises from the motion of the $A-B$ beads against each other.

Using eqs 1 and 2 we can now calculate $\left[G^{\prime}(\omega)\right]$ and $\left[G^{\prime \prime}(\omega)\right]$, the reduced storage and loss moduli of the alternating copolymer ring. Here and in the following we work in reduced units and thus pl ot the results as a function of $\omega \tau_{0}$. In Figure 2, we plot [ $\left.\mathrm{G}^{\prime}(\omega)\right]$ as a function of frequency $\omega$ in double logarithmic scales for a copolymer chain with $\mathrm{N}=500$ repeat unit cells, i.e., $\mathrm{N}_{\text {tot }}$ $=1000$. We vary the parameter $\sigma_{\mathrm{B}}=\zeta_{\mathrm{A}} / \xi_{\mathrm{B}}$ in the range from 0.01 to 100 . The case $\sigma_{B}=1$ (which can be taken as reference) corresponds to the usual, homopolymer Rouse ring. We note that for $\sigma_{\mathrm{B}}=1 \mathrm{eq} 19$ reproduces 


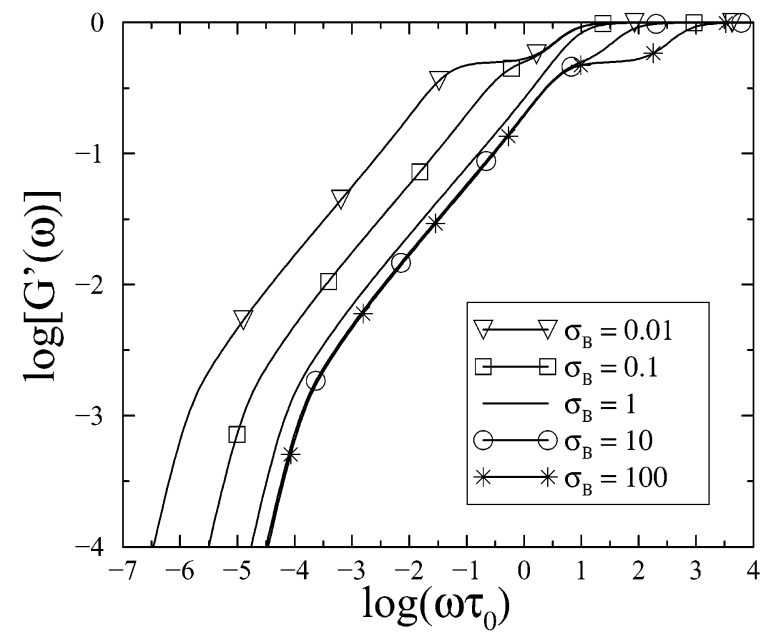

Figure 2. Reduced storage modulus $\left[\mathrm{G}^{\prime}(\omega)\right]$ plotted in double decadic logarithmic scales vs the reduced frequency $\omega \tau_{0}$. Shown are results for an alternating copolymer ring $\left(\mathrm{N}_{\text {tot }}=1000\right)$. The parameter $\sigma_{\mathrm{B}}$ ranges from 0.01 to 100.

the eigenvalues of the Rouse ring. ${ }^{10}$ In this case, $\sigma_{B}=$ 1 , in Figure $2\left[\mathrm{G}^{\prime}(\omega)\right.$ ] displays a power-law behavior, $\left[\mathrm{G}^{\prime}(\omega)\right] \sim \omega^{1 / 2}$ over 4 orders of magnitude in $\omega$, which is bounded by a $\left[\mathrm{G}^{\prime}(\omega)\right] \sim \omega^{2}$ domain at low frequencies and a constant $\left[\mathrm{G}^{\prime}(\omega)\right]$ domain at high frequencies. These domains correspond in the time domain to the so-called terminal and initial ranges, respectively.

Changing $\sigma_{\mathrm{B}}$ from 1 leads to drastic changes in the large $\omega$ - domain. Evident is the appearance of an intermediate plateau for large differences betwen $\xi_{A}$ and $\zeta_{\mathrm{B}}$. For instance, for $\xi_{\mathrm{A}} \gg \xi_{\mathrm{B}}$, i.e., $\sigma_{\mathrm{B}} \gg 1$, the B's are more mobile than the A's and the dynamics starts earlier than in the case of the Rouse chain, composed of A's only. The coresponding typical time $\tilde{\tau}$ is given by $\tau_{0} /\left[4\left(1+\sigma_{\mathrm{B}}\right)\right]$, which for $\sigma_{\mathrm{B}}$ large is approximately $\tau_{0} /$ $\left(4 \sigma_{\mathrm{B}}\right) ; \tilde{\tau}$ decreases with growing $\sigma_{\mathrm{B}}$ and differs strongly from $\tau_{0}$, as may be verified with the help of Figure 2 . The plateau value of $\left[\mathrm{G}^{\prime}(\omega)\right]$ lies at $1 / 2$ as befits the fact that only half of the beads are mobile in this $\omega$-interval . At longer times (smaller $\omega$ ) the behavior of $\left[\mathrm{G}^{\prime}(\omega)\right]$ parallels that of the Rouse-ring, being shifted as a whole toward higher $\omega$ values. For $\zeta_{\mathrm{A}} \ll \zeta_{\mathrm{B}}$, i.e., $\sigma_{\mathrm{B}} \ll 1$ the beads $A$ and $B$ change their roles. This symmetry is evident in Figure 2, where the curves to the values $\sigma_{\mathrm{B}}$ $=\alpha$ and $\sigma_{\mathrm{B}}=1 / \alpha$ have the same shape and are only shifted with respect to one another.

Remarkably, the relaxation spectrum of an alternating copolymer ring leads to even more pronounced effects in the loss modulus [G" $(\omega)$ ]; see Figure 3. Here we pl ot the situation for $\sigma_{\mathrm{B}}=1$ (the Rouse ring), for $\sigma_{\mathrm{B}}$ $=10$ and for $\sigma_{\mathrm{B}}=100$. As usual, all curves scale with $\left[\mathrm{G}^{\prime \prime}(\omega)\right] \sim \omega$ at very small $\omega$ and with $\left[\mathrm{G}^{\prime \prime}(\omega)\right] \sim \omega^{-1}$ at very high $\omega$ values. For $\sigma_{\mathrm{B}}=1$ (the homopolymer case) [G" $(\omega)$ ] shows additionally, as is well-known, the powerlaw Rouse behavior [G" $(\omega)] \sim \omega^{1 / 2}$ on the left-side of the unique maximum. Going now to larger $\sigma_{\mathrm{B}}$ values leads, as is evident from Figure 3, to the appearance of a second maximum at higher frequencies, a feature due to the B's, which have a higher mobility. Evidently, the splitting between the maxima grows with growing $\sigma_{\mathrm{B}}$. The situation for $\sigma_{\mathrm{B}} \ll 1$ is, as before, symmetric, in the sense that the curves corresponding to $\sigma_{\mathrm{B}}=\alpha$ and $\sigma_{\mathrm{B}}=$ $1 / \alpha$ have the same form and are just shifted with respect to each other; we hence dispense from plotting them in Figure 3.

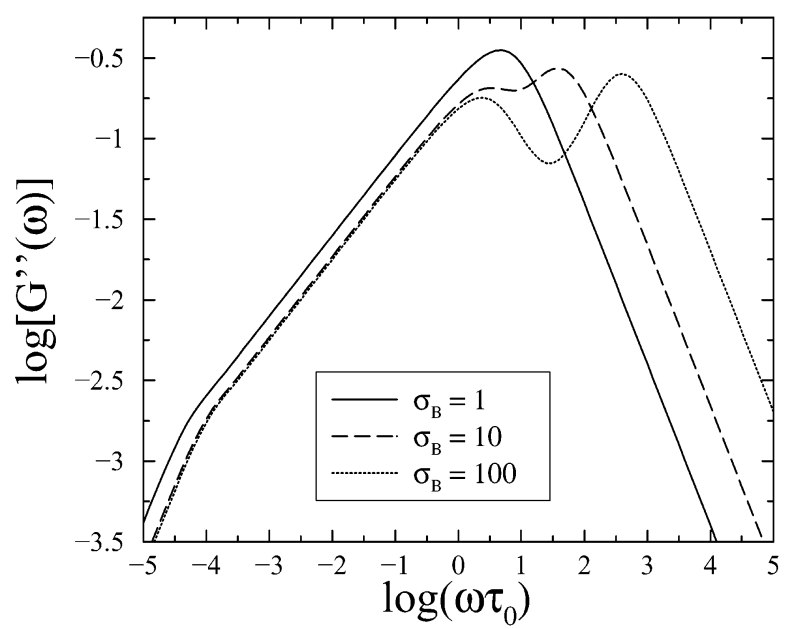

Figure 3. Reduced loss modulus [G" $(\omega)$ ] plotted as in Figure 2. Shown are results for an alternating copolymer ring, $\mathrm{N}_{\text {tot }}=$ 1000. The parameter $\sigma_{\mathrm{B}}$ ranges from 1 to 100 .

We finish this section by considering an alternating copolymer chain with free ends. In this case, we do not have periodic boundary conditions anymore, so that the method developed in the previous section does not hold directly. Since we are interested in the eigenvalues only, we show in the following that those of an alternating -ABAB - copolymer chain with free ends are (apart from a single, special eigenvalue) a subset of those of an alternating $-A B A B-$ copolymer ring with twice more beads. The situation is, in fact, very similar to that of homopolymers, where the eigenvalues for a chain with $\tilde{\mathrm{N}}$ beads are ${ }^{9,10} \lambda_{\text {chain }}=2(1-\cos \mathrm{k})$, with $\mathrm{k}=\pi \mathrm{m} / \tilde{\mathrm{N}}$ and $\mathrm{m}=0,1, \ldots,(\tilde{\mathrm{N}}-1)$. As usual, the eigenvalue $\lambda=$ 0 corresponds to the drift of the chain as a whole. Under the same conditions, a Rouse ring of $2 \tilde{\mathrm{N}}$ beads has as eigenvalues $\lambda_{\text {ring }}=2(1-\cos \mathrm{k})$, with $\mathrm{k}=2 \pi \mathrm{m} / 2 \tilde{\mathrm{N}}=$ $\pi \mathrm{m} / \tilde{\mathrm{N}}$ and $\mathrm{m}=0,1, \ldots,(2 \tilde{\mathrm{N}}-1)$. Thus, the set of eigenvalues $\left\{\lambda_{\text {ring }}\right\}$ has only two nondegenerate eigenvalues, namely, $\lambda=0$ and $\lambda=4$ (the latter corresponds to $\mathrm{k}=\pi)$, whereas all other $(2 \tilde{\mathrm{N}}-2)$ eigenvalues are doubly degenerate, of which thus only $(\tilde{N}-1)$ are distinct. These distinct eigenvalues together with $\lambda=0$ reproduce, in fact, the set of eigenvalues $\left\{\lambda_{\text {chain }}\right\}$ of the Rouse chain.

Now we verify the application of this procedure to the alternating $-A B A B-$ chain with free ends; i.e., we compare its eigenvalues to those of an alternating ring of $2 \mathrm{~N}$ subunits (i.e., having $4 \mathrm{~N}$ beads). For such a ring the spectrum is given by eq 19 , now with

$$
\mathrm{k}_{1}=\pi \mathrm{m}_{1} / \mathrm{N}
$$

and $m_{1}=0,1, \ldots,(2 \mathrm{~N}-1)$. It is clear from the general approach of section 3 that all normal modes of a system consisting of repeat unit cells can be classified into two groups: (i) modes involving the motions of cells against each other $\left(k_{1} \neq 0\right)$ and (ii) modes without such motions $\left(k_{1}=0\right)$. As before, in the homopolymer case, the class (i) modes are double degenerated, and each of them appears exactly once in the spectrum of the alternating chain with free ends. Moreover, the eigenvalue $\lambda=0$ (related to the drift of the macromol ecule) also appears both in the alternating chain and in the twice-as-large ring. We now claim that the last eigenvalue for the alternating copolymer chain with free ends equals $\lambda_{1}=$ $\left(1+\sigma_{\mathrm{B}}\right)$. That this is indeed the case can be seen readily; 


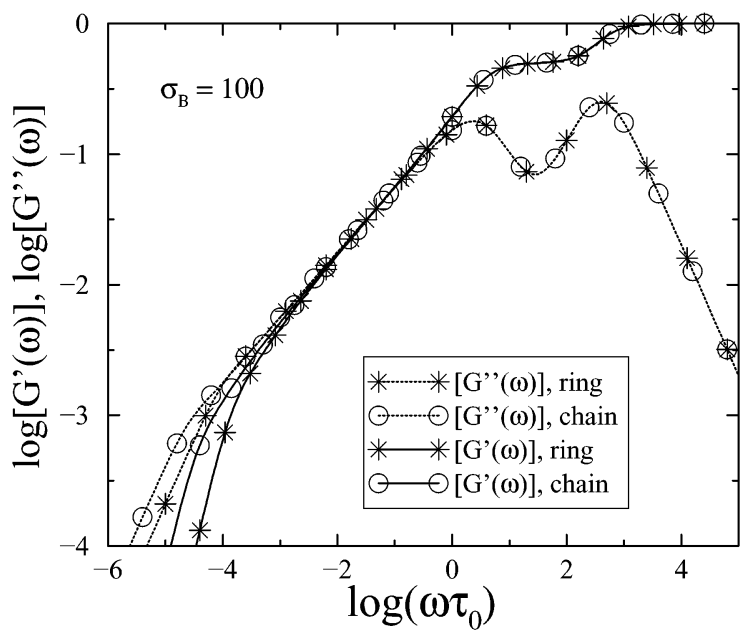

Figure 4. Reduced storage $\left[\mathrm{G}^{\prime}(\omega)\right]$ and loss $\left[\mathrm{G}^{\prime \prime}(\omega)\right]$ moduli plotted as in Figure 2. Shown are results for a copolymer ring and for a copolymer chain with free ends, both for $\sigma_{\mathrm{B}}=100$ and $\mathrm{N}_{\text {tot }}=1000$.

one has only to verify by insertion that taking the position vectors $\mathbf{R}_{\mathrm{A \alpha}}(\mathrm{t})$ and $\mathbf{R}_{\mathrm{B} \alpha}(\mathrm{t})$ for the $\mathrm{A}$ and $\mathrm{B}$ beads in the $\alpha$ th unit cell in the form

$$
\mathbf{R}_{\mathbf{A} \alpha}(\mathrm{t})=\mathbf{C}(-1)^{\alpha}\left(\sigma_{\mathrm{B}}\right)^{\alpha} \exp \left(-\lambda_{1} \mathrm{t} / \tau_{0}\right)
$$

and

$$
\mathbf{R}_{\mathbf{B} \alpha}(\mathrm{t})=\mathbf{C}(-1)^{\alpha+1}\left(\sigma_{\mathrm{B}}\right)^{\alpha+1} \exp \left(-\lambda_{1} \mathrm{t} / \tau_{0}\right)
$$

(with $\mathbf{C}$ being a constant) fulfills the corresponding Langevin equation when $\lambda_{1}=\left(1+\sigma_{\mathrm{B}}\right)$. As a side remark, we note that $\lambda_{1}$ for the alternating copolymer chain does not (for $\sigma_{\mathrm{B}} \neq 1$ ) correspond to any of the three nonvanishing, nondegenerate eigenavalues of the twice-aslarge ring; this eigenvalue is sui generis. In the limit $\sigma_{\mathrm{B}} \rightarrow 1$ one has $\lambda_{1}=2$, a legitimate eigenvalue of the homopolymer chain, which appeared there from a double degenerate eigenvalue of the corresponding homopolymer ring. We note that we have verified our findings through the direct numerical diagonalization of $\tilde{\mathbf{A}}$ matrices for several copolymer chains (up to $\mathrm{N}_{\text {tot }}=$ 500) and for their corresponding rings; all the results obtained confirm that the above analysis is correct. We also have reproduced the findings of ref 12 (their case IIIa) for $\sigma_{\mathrm{B}}=185$.

We are now in the position to compare the moduli $\left[\mathrm{G}^{\prime}(\omega)\right]$ and $\left[\mathrm{G}^{\prime \prime}(\omega)\right]$ for an alternating copolymer chain to those for a ring with the same number of beads. In Figure 4, we plot [ $\left.\mathrm{G}^{\prime}(\omega)\right]$ and [G"$\left.(\omega)\right]$, both for the chain and for the ring, taking $\mathrm{N}=500\left(\mathrm{~N}_{\text {tot }}=1000\right)$ and $\sigma_{\mathrm{B}}=$ 100. We notice small differences in the domain of very small $\omega$ (i.e., at long times), close to the domain of the terminal relaxation. These differences are due to differences in the maximal relaxation times. For alternating copolymers the minimal, nonzero eigenvalue $\lambda_{\min }$ can be estimated (by expanding eq 19 for small $k_{1}$ ) to be:

$$
\lambda_{\min } \simeq \frac{\sigma_{\mathrm{B}}}{\left(1+\sigma_{\mathrm{B}}\right)} \mathrm{k}_{\min }^{2}
$$

where $\mathrm{k}_{\min }$ is $2 \pi / \mathrm{N}$ for the ring and $\pi / \mathrm{N}$ for the chain, compare eq 14 and 20. Hence, at an equal number of beads, for a ring $\lambda_{\min }$ is around four times larger than for the chain, or written differently, one has $\tau_{\max }^{\text {(chain)/ }}$ $\tau_{\max }^{(\text {ring })} \cong 4$, where $\tau_{\max }^{(\text {chain) }}$ and $\tau_{\max }^{\text {(ring) }}$ are the maximal relaxation times of the chain and of the ring, respectively. This explains closely the differences in [G' $(\omega)]$ and [ $\left.\mathrm{G}^{\prime \prime}(\omega)\right]$ at small $\omega$ between the rings and the chains; see F igure 4. We emphasize that these differences occur at very low frequencies, i.e., very far from the initial relaxation domain. Therefore, all conclusions drawn in this domain for the copolymer ring, see Figures 2 and 3 , also hold for the corresponding copolymer chain.

\section{Cross-Linked, Alternating Copolymers}

Now we turn to the viscoelastic dynamic properties of end-linked networks built from $-A B A B$ - alternating copolymer chains. Exemplarily, we will study twodimensional square lattices made from such chains; toward the end of the section we will remark on how different types of Iattices affect the results obtained. In our model we let (see Figure 1) the friction constants $\sigma_{\mathrm{C}}$ of the junctions (network vertexes) differ from those of the $A$ and $B$ beads, since in many applications such many-functional points are realized by beads of a different kind. ${ }^{31,48}$ This means that we now have (apart from $\sigma_{\mathrm{A}}=1$ ) two parameters, namely $\sigma_{\mathrm{B}}$ and $\sigma_{\mathrm{C}}$. In fact, we differentiate here between two types of effects, one being related to the behavior of the copolymer chains between network junctions, the other type involving the motion of the junctions.

We start by focusing on the role of the network junctions; for this, we vary $\sigma_{\mathrm{C}}=\xi_{\mathrm{A}} / \xi_{\mathrm{C}}$, while setting $\sigma_{\mathrm{B}}$ $=1$. At first we note that one of us already considered the relaxation properties of Rouse chains cross-linked into regular lattices. ${ }^{49-53}$ Thus, for $\sigma_{\mathrm{C}}=2$ in the case of the square lattice and $\sigma_{\mathrm{C}}=3$ for the cubic lattice, the relaxation spectrum can be obtained analytically; furthermore, no significant differences appeared in the numerical study for $\sigma_{\mathrm{C}}=1$ for the same lattices. ${ }^{6,7}$ The basic aspect of regular networks built from Rouse chains is that their relaxation spectrum displays two characteristic domains; these correspond to the small-scale (intrachain) motions and to the large-scale (interchain) relaxation of the network, respectively. The intrachain part of the spectrum reproduces closely the spectrum of single Rouse chains, ${ }^{9,10}$ i.e., one observes a $\left[\mathrm{G}^{\prime}(\omega)\right] \sim$ $\omega^{1 / 2}$ behavior in this domain. At lower frequencies (corresponding to the larger-scale motions), [G' $(\omega)]$ crosses over to a $\left[\mathrm{G}^{\prime}(\omega)\right] \sim \omega^{\alpha}$ behavior, where $\alpha$ depends on the lattice type considered. In fact $\alpha=\mathrm{d} / 2$, where $\mathrm{d}$ is the lattice dimension; hence, $\alpha$ equals $3 / 2$ for three dimensional ${ }^{52-54}$ and 1 for two-dimensional ${ }^{49-51,54}$ lattices. We note that for these networks the maximal relaxation time of the Rouse chains connecting the vertexes and the minimal relaxation time of the lattice were found to be equal. $49,50,52,53$ This leads to a very smooth behavior of $\left[\mathrm{G}^{\prime}(\omega)\right]$ and $\left[\mathrm{G}^{\prime \prime}(\omega)\right]$ in the region intermediate between the domains of intrachain and of interchain relaxation.

Now we return to the case of general $\sigma_{\mathrm{C}}$, while still keeping $\sigma_{\mathrm{A}}=\sigma_{\mathrm{B}}=1$ fixed. Using the methods of section 3 , we calculate $\left[\mathrm{G}^{\prime}(\omega)\right]$ and $\left[\mathrm{G}^{\prime \prime}(\omega)\right]$, for a square lattice of $20 \times 20$ unit cells, built from Rouse - ABAB - chains of 100 beads, by evaluating the corresponding eigenvalues. For this, we vary $\sigma_{\mathrm{C}}$. The case $\sigma_{\mathrm{C}}<1$ corresponds to having the friction constant $\xi_{C}$ of the junctions larger than that $\left(\xi_{A}=\zeta_{B}\right)$ of the beads of the chains. This affects strongly, especially for very small $\sigma_{C}$, the mechanical relaxation of the network in the domain 


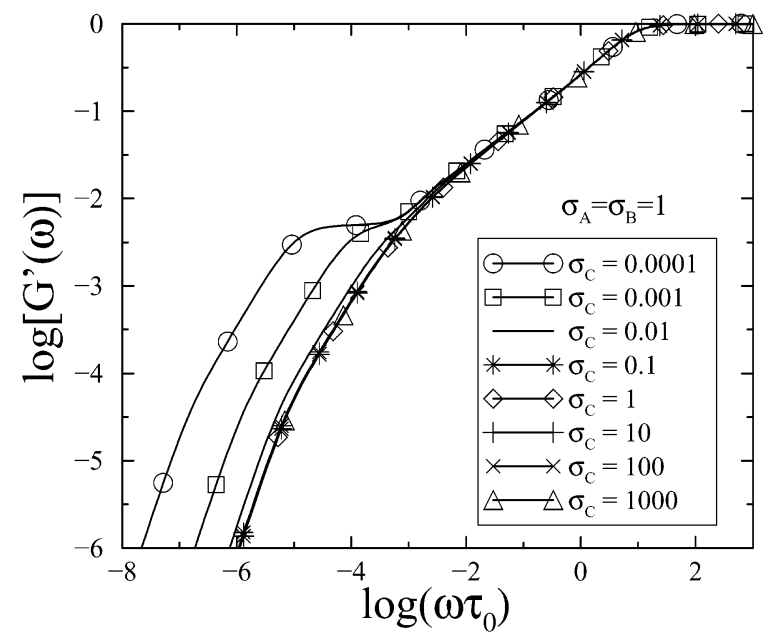

Figure 5. Reduced storage modulus $\left[\mathrm{G}^{\prime}(\omega)\right]$ plotted as in Figure 2. Shown are results for al ternating copolymers (of 100 beads each) cross-linked into a $20 \times 20$ square lattice. Here $\sigma_{\mathrm{A}}=\sigma_{\mathrm{B}}=1$, and $\sigma_{\mathrm{C}}$ ranges from 0.0001 to 10000 ; see text for details.

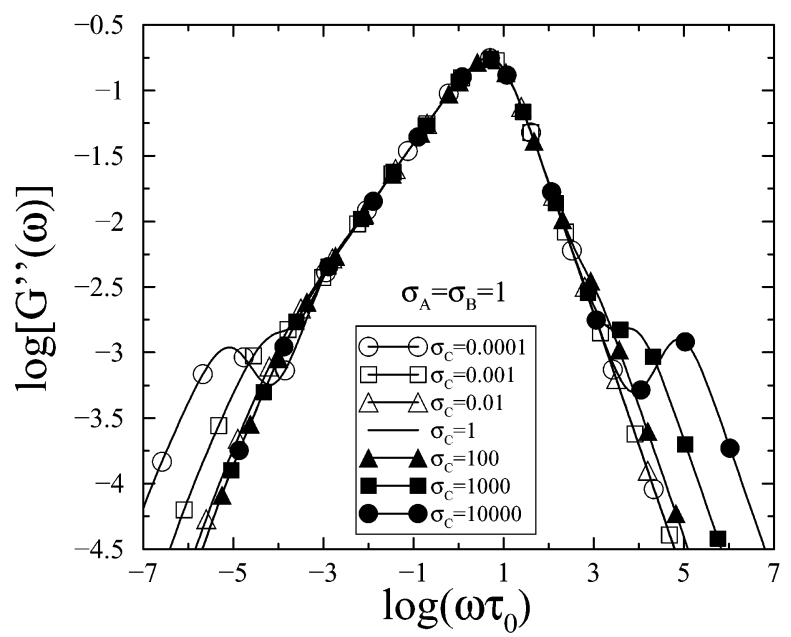

Figure 6. Reduced loss modulus [G" $(\omega)$ ] plotted as in Figure 2 , for the same system as in Figure 5 . Here $\sigma_{\mathrm{C}}$ ranges from 0.0001 to 10000 .

intermediate between the intrachain and the purely lattice relaxation. In Figure 5, we even observe for $\sigma_{\mathrm{C}}$ $=0.001$ and for $\sigma_{\mathrm{C}}=0.0001$ the appearance of plateau in $\left[\mathrm{G}^{\prime}(\omega)\right.$ ]. Physically, this is due to the separation of the scales of motion of the network chains and of the junctions, which, for $\sigma_{\mathrm{C}} \ll 1$, leads to a large gap in the relaxation spectrum. We note that such a scale-separation is very important when modeling the relaxation of polymer networks analytically. ${ }^{55}$

For $\sigma_{\mathrm{C}}>1$ the friction constant of the junctions, $\xi_{\mathrm{C}}$, is less than $\xi_{A}=\xi_{B}$. Nonetheless, the junctions preserve the interchain connectivity. According to Figure 5, taking $\sigma_{\mathrm{C}} \gg 1$ instead of $\sigma_{\mathrm{C}}=1$ does not affect the network's dynamics in the intermediate frequency domain. Evident changes are in the low-frequency domain, where the terminal relaxation is shifted to higher frequencies. Furthermore, there appears an additional plateau at high-frequencies, not resolved in Figure 5, and probably also of difficult experimental access. Similar effects can be observed in the loss modulus [G" $(\omega)$ ]; see Figure 6 . As mentioned, for $\zeta_{C}$ highly different from $\xi_{A}=\xi_{B}$ one has a gap in the eigenvalues' spectrum. This is reflected for $\sigma_{\mathrm{C}}=0.0001$

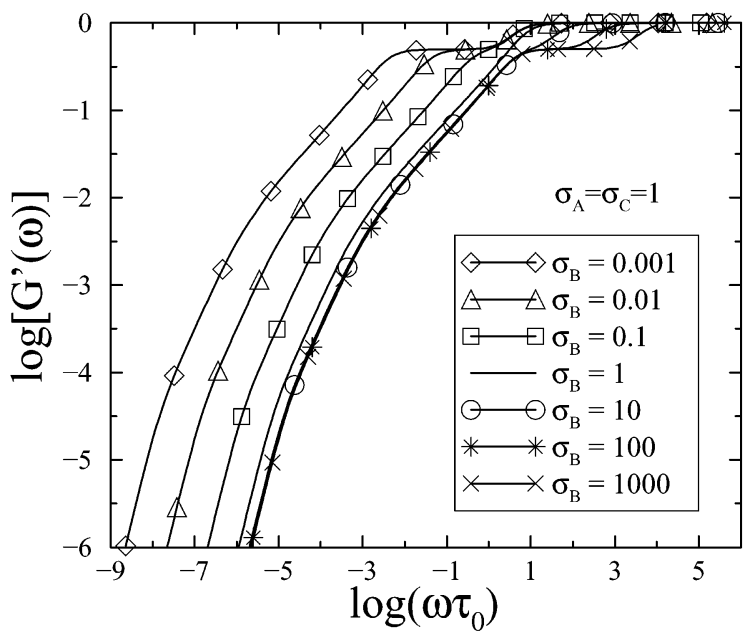

Figure 7. Reduced storage modulus $\left[\mathrm{G}^{\prime}(\omega)\right]$ plotted as in Figure 2. The same setup is used as in Figure 5 , only that now $\sigma_{\mathrm{A}}=\sigma_{\mathrm{C}}=1$ and $\sigma_{\mathrm{B}}$ ranges from 0.001 to 1000 .

by the appearance in $\left[\mathrm{G}^{\prime \prime}(\omega)\right]$ of an additional peak on the small- $\omega$ side, when compared to [G" $(\omega)]$ for $\sigma_{\mathrm{C}}=1$. In symmetrical fashion, an additional peak appears for $\sigma_{\mathrm{C}}=10000 \mathrm{in}\left[\mathrm{G}^{\prime \prime}(\omega)\right]$ on the large $\omega$ side.

As a second step in the analysis we set $\xi_{C}=\xi_{A}$, i.e., $\sigma_{\mathrm{C}}=1$, and study the role of the $\mathrm{B}$ beads on the network dynamics. We hence vary $\sigma_{\mathrm{B}}$. In Figure 7 we plot our numerical results for the reduced storage modulus [G' $(\omega)$ ] of a square lattice with $20 \times 20$ junctions, built from alternating copolymer chains of 100 beads each. As was to be expected, in the high-frequency domain we observe the appearance of plateaus, both for $\sigma_{\mathrm{B}} \ll 1$ and for $\sigma_{\mathrm{B}} \gg 1$, whose widths depend on the mismatch between $\zeta_{A}$ and $\xi_{B}$; these results may be compared to the findings in Figure 2. A major difference appears now only in the very low- frequency region, before the $\omega^{1 / 2}$ chainlike behavior; we have there $\mathrm{a}\left[\mathrm{G}^{\prime}(\omega)\right] \sim \omega$ dependence, typical for a 2D lattice. Moreover, we note that for a 3D regular structure ${ }^{52,53}$ the power-law would be $\omega^{3 / 2}$. This allows us to consider how far our results are influenced by the choice of the lattice. It turns out that the influence of different lattices is mainly felt in the low-frequency domain of $\left[\mathrm{G}^{\prime}(\omega)\right]$ and $\left[\mathrm{G}^{\prime \prime}(\omega)\right]$, where both quantities scale as $\omega^{\mathrm{d} / 2}$, $\mathrm{d}$ being the dimensionality of the lattice. The special aspect considered by us here, namely the fact of having alternating copolymers, becomes evident at much higher frequencies (i.e., at shorter times) and is hence not influenced by the type of lattice chosen for modeling the network.

As the final step of the analysis, we take now both $\sigma_{\mathrm{B}}$ and also $\sigma_{\mathrm{C}}$ different from unity. In Figure 8, we plot the storage modulus, [G' $(\omega)]$, and in Figure 9, the loss modulus, [G" $(\omega)$ ], of a square network built from alternating copolymer chains. Here we take $\zeta_{A}, \zeta_{B}$, and $\xi_{\mathrm{C}}$ to be very different, such that $\sigma_{\mathrm{B}}=100$ and $\sigma_{\mathrm{C}}=$ 0.0001 . This leads for $\left[\mathrm{G}^{\prime}(\omega)\right]$ to two plateaus, as may be seen from Figure 8. To be able to unravel their nature we plot in Figure 8 (and also in Figure 9), for comparison, the situation for a single alternating copolymer chain. This allows us to relate the plateau at higher $\omega$ values to the gap in the relaxation spectrum of the alternating copolymer. The second plateau, at low frequencies, is hence due to the gap in eigenvalues in the region intermediate between the relaxation inside the chains and the relaxation involving the whole lattice. Thus, we observe here three characteristic 


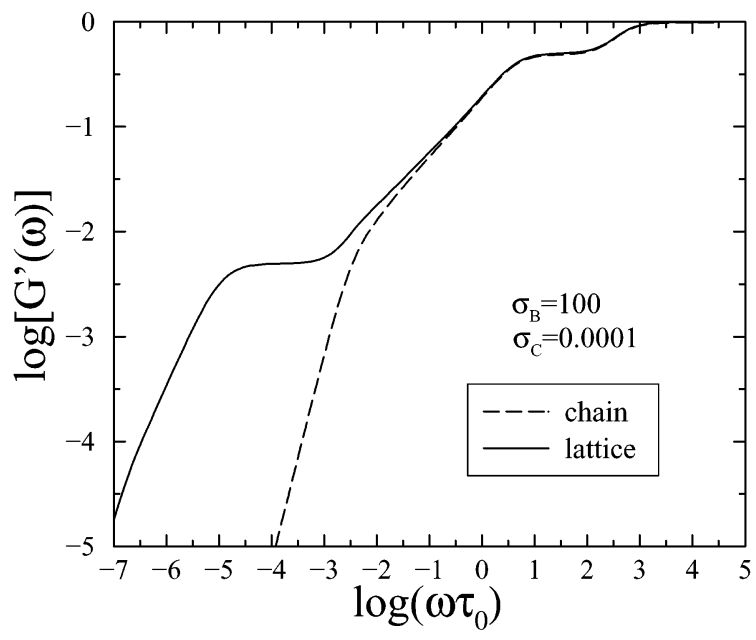

Figure 8. Reduced storage modulus $\left[\mathrm{G}^{\prime}(\omega)\right]$ plotted as in Figure 2. The situation is as in Figure 5 , only that now $\sigma_{\mathrm{A}}=$ $1, \sigma_{\mathrm{B}}=100$, and $\sigma_{\mathrm{C}}=0.0001$. Through the dashed line, we indicate $\left[\mathrm{G}^{\prime}(\omega)\right]$ for a single alternating copolymer chain consisting of 100 beads.

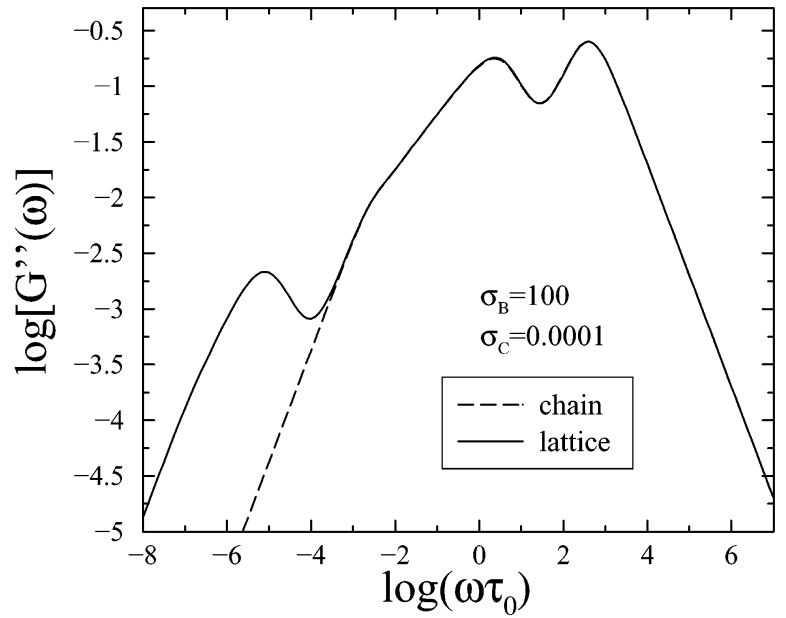

Figure 9. Reduced loss modulus [G" $(\omega)]$ plotted as in Figure 2 for the same systems as in Figure 8.

regions of relaxation, separated by plateaus. Indeed, the corresponding $\left[\mathrm{G}^{\prime \prime}(\omega)\right]$ displays under the same conditions three peaks; see Figure 9.

\section{Conclusions}

In this paper, we presented a theoretical study of cross-linked copolymers and model ed them in the frame work of generalized Gaussian structures (GGS), with the added feature that beads of different kinds have also different friction constants. The model consisted of copolymer chains cross-linked into topologically regular lattices. We devel oped a general method for determining the spectrum of relaxation times for such networks, a direct extension of the approach previously developed by us for homopolymer networks. ${ }^{6-8}$

As an example, we applied this general approach to polymer networks built from evenly alternating copolymer chains containing two kinds of beads. At first, we focused on un-cross-linked polymer rings and determined their relaxation spectrum analytically. We found that when the difference between the friction constants of the beads of two kinds $\left(\sigma_{B}=\zeta_{A} / \xi_{B}\right)$ is sufficiently large, in the large $\omega$-region the reduced storage (shear) modulus [ $\left.G^{\prime}(\omega)\right]$ of the copolymer deviates strongly from the behavior typical for homopolymers: We found that [G' $(\omega)$ ] shows a plateau-type behavior in this domain, due to a large gap in the mobilities of the $A$ and of the B components. Even more pronounced effects are re vealed by $\left[G^{\prime \prime}(\omega)\right]$ where this large difference in mobilities leads to the appearance of an additional peak.

Cross-linking such copolymer chains into regular lattices leads to additional features in the storage and loss moduli at low-frequencies, where modes due to the lattice lead to a $\omega^{\mathrm{d} / 2}$ scaling behavior. Remarkably, the most interesting situation occurs when all three kinds of beads (the A's, the B's, and the junctions) have vastly different mobilities. The storage modulus [ $\mathrm{G}^{\prime}(\omega)$ ] of such networks has a very structured behavior: One observes three rel axation regions separated by two plateaus. The first plateau is in the high-frequency domain and corresponds to the (above-mentioned) gap in the relaxation spectrum of alternating copolymer chains, while the second plateau is in the intermediate frequency domain and separates the intrachain relaxation from the lattice relaxation. Moreover, in this case the loss modulus [G" $(\omega)$ ] shows three peaks. We infer that such differences in the mobilities of chemically different species in cross-linked alternating copolymers could be readily detected through appropriate mechanical relaxation experiments.

Considering extensions to more realistic systems one may include hydrodynamic interactions and also take excluded-volume effects into account. Staying in the framework of GGS, one may also consider fluctuations in the chemical regularity (i.e., use of different beads to account for different segment lengths, enlargement of the repeat patterns of the unit cells, etc.) We defer such extensions to subsequent works.

Acknowledgment. This work was supported by the Fonds der Chemischen I ndustrie, by BMBF, and by the DFG. A.A.G. acknowledges the support of the Alexander von Humboldt Foundation, of the INTAS (Grants 00712 and 99-1114), of the Russian Foundation of Basic Research (Grant 02-03-33132), of the Russian Federal Program "Integration" (Grant 32638), and of the ESF Scientific Program SUPERNET.

\section{References and Notes}

(1) Sommer, J .-U.; Blumen, A. J . Phys. A 1995, 28, 6669.

(2) Schiessel, H. Phys. Rev. E 1998, 57, R5775.

(3) Friedrich, Ch.; Schiessel, H.; Blumen, A. In Advances in the Flow and Rheology of Non-Newtonian Fluids; Siginer, D. A., DeKee, D., Chhabra, R. P., Eds.; Elsevier: Amsterdam, 1999; p 429.

(4) Schiessel, H.; Friedrich, Ch.; Blumen, A. In Applications of Fractional Calculus in Physics; Hilfer, R., Ed.; World Scientific: Singapore, 2000; p 331.

(5) Blumen, A.; Gurtovenko, A. A.; J espersen, S. J . Non-Cryst. Solids 2002, 305, 71

(6) Gurtovenko, A. A.; A. Blumen, A. J . Chem. Phys. 2001, 115, 4924.

(7) Gurtovenko, A. A.; Blumen, A. Macromolecules 2002, 35, 3288.

(8) Gurtovenko, A. A.; Gotlib, Yu. Ya.; Blumen, A. Macromolecules 2002, 35, 7481.

(9) Rouse, P. E. J . Chem. Phys. 1953, 21, 1272

(10) Doi, M.; Edwards, S. F. The Theory of Polymer Dynamics; Clarendon: Oxford, England, 1986.

(11) Grosberg, A. Yu.; Khokhlov, A. R. Statistical Physics of Macromolecules; AIP Press: New York, 1994.

(12) Hansen, D. R.; Shen, M. Macromolecules 1975, 8, 343

(13) Hall, W. F.; De Wames, R. E. Macromolecules 1975, 8, 349.

(14) Stockmayer, W. H.; Kennedy, J . W. Macromolecules 1975, 8, 351.

(15) Wang, F. W.; DiMarzio, E. A. Macromolecules 1975, 8, 356. 
(16) Wang, F. W. Macromolecules 1975, 8, 364

(17) Man, V. F.; Schrag, J. L.; Lodge, T. P. Macromolecules 1991, $24,3666$.

(18) Zimm, B. J . Chem. Phys. 1956, 24, 269.

(19) Fredrickson, G. H.; Bates, F. S. Annu. Rev. Mater. Sci. 1996, $26,501$.

(20) Hamley, I. M. The Physics of Block Copolymers; Oxford University Press: Oxford, England, 1998.

(21) Lay, S.; Sommer, J .-U.; Blumen, A. J . Chem. Phys. 1999, 110, 12173.

(22) Shen, M.; Hansen, D. R. J . Polym. Sci., Part C 1974, 46, 55.

(23) Hansen, D. R.; Shen, M. Macromolecules 1975, 8, 903.

(24) Krause, S. In Block and Graft Polymers; Burke, J . J ., Weiss, V., Eds.; Syracuse University Press: Syracuse, NY, 1973; $p$ 143.

(25) Robeson, L. M.; Matzner, M.; Fetters, L. J .; McGrath, J. E. In Recent Advances in Polymer Blends, Grafts, and Blocks; Sperling, L. H., Ed.; Plenum Press: New York, 1974; p 281.

(26) Han, C. D.; Baek, D. M.; Kim, J. K.; Hashimoto, T.; Okamoto S. Macromolecules 1991, 24, 5408.

(27) Utracki, L. A.; Simha, R. Macromolecules 1968, 1, 505.

(28) Ohnumba, H.; Kotaka, T.; I nagaki, H. Polym. J . 1970, 1, 716.

(29) Sato, T.; Watanabe, H.; Osaki, K. Macromol ecules 1996, 29, 6231.

(30) Kleppinger, R.; Mischenko, N.; Theunissen, E.; Reynaers, H L.; Koch, M. H. J .; Almdal, K.; Mortensen, K. Macromol ecules 1997, 30, 7012

(31) Daniel, C.; J ao, T.-C.; Bauer, B. J .; Amis, E. J . Macromolecules 1999, 32, 8938.

(32) Hedrick, J. L.; B. Haidar, B.; Russell, T. P.; Hofer, D. C. Macromolecules 1988, 21, 1967.

(33) Markoski, L. J .; Walker, K. A.; Deeter, G. A.; Spilman, G. E.; Martin, D. C.; Moore, J. S. Chem. Mater. 1993, 5, 248.

(34) Westermann, S.; Urban, V.; Pyckhout-Hintzen, W.; Richter, D.; Straube, E. Macromolecules 1996, 29, 6165.

(35) Szuromi, E.; Berka, M.; Borbély, J . Macromol ecules 2000, 33, 3993.

(36) Khanna, Y. P.; Sibilia, J . P.; Chandrasekaran, S. Macromolecules 1986, 19, 2426.
(37) Chu, B.; Wu, C. Macromolecules 1987, 20, 93.

(38) Hirabayashi, T.; Yamauchi, K.; Yokota, K. Macromolecules 1990, 23, 935; 1991, 24, 4543.

(39) Wang, Z.; Tontisakis, A.; Tuminello, W. H.; Buck, W.; Chu, B. Macromolecules 1990, 23, 1444

(40) Konzelman, J .; Wagener, K. B. Macromolecules 1996, 29, 7657.

(41) Interrante, L. V.; Shen, Q.; Li, J . Macromolecules 2001, 34 1545.

(42) Wagner, Z. R.; Roenigk, T. K.; Goodson, F. E. Macromolecules 2001, 34, 5740 .

(43) Ferry, J . D. Viscoelastic Properties of Polymers, 3rd ed.; J Wiley \& Sons: New York, 1980

(44) Biswas, P.; Kant, R.; Blumen, A. Macromol. Theory Simul. 2000, 9, 56

(45) Kittel, C. Introduction to Sol id State Physics, 6th ed.; J . Wiley \& Sons: New York, 1986

(46) Ashcroft, N. W.; Mermin, N. D. Solid StatePhysics; Sanders College: Philadel phia, PA, 1981.

(47) Ziman, J. M. Principles of the Theory of Solids, 2nd ed.; Cambridge University Press: Cambridge, England, 1972.

(48) Okabe, M.; Mitsui, K. K.; Uranaka, H.; Takahashi, M.; Matsuda, M. Polym. J . 1992, 24, 653.

(49) Gotlib, Yu. Ya.; Gurtovenko, A. A. Macromol. Theory Simul. 2000, 9, 407.

(50) Gurtovenko, A. A.; Gotlib, Yu. Ya. Macromol. Theory Simul. 2000, 9, 416.

(51) Chompff, A. J .; Duiser, J . A. J . Chem. Phys. 1966, 45, 1505.

(52) Gurtovenko, A. A.; Gotlib, Yu. Ya. Macromolecules 1998, 31 5756.

(53) Gurtovenko, A. A.; Gotlib, Yu. Ya. Macromolecules 2000, 33, 6578.

(54) Gurtovenko, A. A.; Gotlib, Yu. Ya. J . Chem. Phys. 2001, 115, 6785.

(55) Sommer, J.-U.J . Chem. Phys. 1991, 95, 1316.

MA0214409 